\title{
EIGENVALUES OF THE LAPLACE-BELTRAMI OPERATOR ON A LARGE SPHERICAL CAP UNDER THE ROBIN PROBLEM
}

\author{
Yoshitsugu Kabeya*, TATSUKi KaWAKAmi ${ }^{\dagger}$, \\ Atsushi Kosaka AND HirokazU NinOMIYA
}

\begin{abstract}
Eigenvalues of the Laplace-Beltrami operator on a spherical cap is considered under the homogeneous Robin condition. The asymptotic behavior of eigenvalues and the influence of the eigenvalues by the boundary conditions are discussed as the cap becomes large so that the domain covers almost the whole sphere.
\end{abstract}

\section{Introduction}

In this paper we study the linear eigenvalue problem

$$
\begin{cases}\Lambda v+\lambda v=0 & \text { in } \Omega_{\varepsilon} \subset \mathbf{S}^{n}, \\ (\cos \sigma) \partial_{\mathbf{n}} v+(\sin \sigma) v=0 & \text { on } \partial \Omega_{\varepsilon},\end{cases}
$$

where $\Lambda$ is the Laplace-Beltrami operator on the unit sphere $\mathbf{S}^{n}\left(\subset \mathbf{R}^{n+1}\right), n \geq 2$, $\Omega_{\varepsilon}$ is the spherical cap centered at the North Pole $(0,0, \ldots, 0,1)$ of its geodesic radius $\pi-\varepsilon, \varepsilon>0, \partial_{\mathbf{n}}$ is the derivative in the direction of the outer unit normal to $\partial \Omega_{\varepsilon}, \sigma \in[0, \pi / 2]$ and $\lambda>0$ are parameters. We investigate the behavior of the eigenvalues of $-\Lambda$ as $\varepsilon \rightarrow+0$. The investigation of the behaviors of the eigenvalues is fundamental and important. Once we know the exact information on the eigenvalues, we can consider the nonlinear elliptic problem

$$
\begin{cases}\Lambda u+\lambda u+|u|^{p-1} u=0 & \text { in } \Omega_{\varepsilon} \subset \mathbf{S}^{n} \\ (\cos \sigma) \partial_{\mathbf{n}} u+(\sin \sigma) u=0 & \text { on } \partial \Omega_{\varepsilon},\end{cases}
$$

* Supported in part by Grant-in-Aid for Scientific Research (C) (No. 23540248), Japan Society for the Promotion of Science.

${ }^{\dagger}$ Supported in part by Grant-in-Aid for Young Scientists (B) (No. 24740107), Japan Society for the Promotion of Science.

\Supported in part by a Research Project Grant (A) from the Institute of Science and Technology, Meiji University and Grant-in-Aid for Challenging Exploratory Research (No. 23654042, 25610036), Japan Society for the Promotion of Science.

2010 Mathematics Subject Classification. Primary 35J05, Secondary 35J25, 33C55.

Key words and phrases. spherical cap, eigenvalues, the associated Legendre functions.

Received April 1, 2014; revised April 30, 2014. 
and discuss the local bifurcation from the trivial solution $u \equiv 0$ in a forthcoming paper.

In the closely related papers by Bandle, the first and the last authors of this paper [6] and by the third author [11], the distribution of the eigenvalues of the Laplace-Betrami operator was discussed. In [6], (1.1) under the Neumann condition $(\sigma=0)$ was considered and in [11] the two dimensional Dirichlet case $(\sigma=\pi / 2)$ was done. In this paper, we focus on the effect of the presence of the parameter $\sigma$. It is plausible that the dependence of the eigenvalues on $\sigma$ is continuous, however, how is the influence of $\sigma$ ?

As for the pioneering works on the dependence of the eigenvalues concerning the domain perturbation, there are results due to Ozawa [16, 17, 18, 19]. In [17], the relationship between the capacity of the small domain which was rid out from the fixed domain and the behavior of the eigenvalues was discussed. On manifolds under the Dirichlet boundary condition, the estimates of the perturbed eigenvalues were obtained by Courtois [10] also in the context of the capacity. Some results for simple eigenvalues in planar domains with holes under the Neumann condition are found in Lanza de Cristoforis [12].

In recent years such problems have been investigated on more general Riemannian manifolds. Results for radial solutions in geodesic balls in spaces of constant curvature (spheres or a domain on the hyperbolic space) are found in Bandle, Brillard and Flucher [4], Bandle and Peletier [7], Bandle and Benguria [3], Brezis and Peletier [9] and the references quoted therein.

We shall treat problem $(1.1)$ as a perturbation in $\varepsilon$ of the problem

$$
\Lambda u+\lambda u=0 \quad \text { in } \mathbf{S}^{n} .
$$

In this case, all the spectra are completely understood. In fact (cf. i.e. Shimakura [21]) the $k$-th eigenvalue (counting from zero) of $-\Lambda$ on $\mathbf{S}^{n}$ is

$$
k(k+n-1)
$$

and its multiplicity is

$$
(2 k+n-1) \frac{(k+n-2) !}{(n-1) ! k !} .
$$

The eigenfunctions are expressed in terms of the associated Legendre polynomials. In case of $k=0$, the constant 1 is the corresponding eigenfunction.

This paper is devoted to study of the asymptotic behavior of the eigenvalues in $\Omega_{\varepsilon}$ for small $\varepsilon$. Concerning the Neumann boundary condition, the detailed properties of the associated Legendre functions or the Gauss hypergeometric functions are used in [6]. As for the Dirichlet problem, the third author of this paper [11] also investigated the behavior of eigenvalues and showed their asymptotic behavior in the case of $n=2$ by using the knowledge of the zeros of the associated Legendre functions as discussed in Baginski [1, 2] and Macdonald [13].

In contrast to the Dirichlet case or the Neumann case, very little is known for the Robin problem. Although we can consider (1.1) in any dimension which 
is greater than one, however, we give here results in the cases $n=2$ and $n=3$. General cases will be discussed in a forthcoming paper.

The main results are as follows.

TheORem 1.1. Let $n=2$. Fix $\sigma \in(0, \pi / 2)$. For any $k \in \mathbf{N}$, there exist $(k+1)$ distinct eigenvalues $\lambda_{\varepsilon, k, m, \sigma}(m=0,1, \ldots, k)$ of $-\Lambda$ exist near $k(k+1)$. As $\varepsilon \rightarrow+0$, the following asymptotic expansion hold:

$$
\lambda_{\varepsilon, k, m, \sigma}-k(k+1)=c_{k, m, \sigma, 2} \varepsilon^{\max \{2 m, 1\}}+o\left(\varepsilon^{\max \{2 m, 1\}}\right) \quad(m=0,1,2, \ldots, k),
$$

where

$$
c_{k, m, \sigma, 2}= \begin{cases}-\frac{(2 k+1)(m+k) !}{4^{m}(k-m) ! m !(m-1) !}, & m=1,2,3, \ldots, k \\ \frac{2 k+1}{2} \tan \sigma, & m=0 .\end{cases}
$$

Moreover, the multiplicity of $\lambda_{\varepsilon, k, m, \sigma}$ is 2 if $m \geq 1$ and that of $\lambda_{\varepsilon, k, 0, \sigma}$ is 1 .

Remark 1.1. In $n=2$ case, if $m \geq 1$, then the leading term is exactly the same as that obtained in [6] under the Neumann condition. However, if $m=0$, the difference from the Neumann condition case appears since $\tan \sigma=0$. In this case, the order is $\varepsilon^{2}$ by [6].

The relationship between the Dirichlet case and the Neumann case will be seen in (3.17) in Section 3. We can deduce the same order and the same coefficient as those obtained in [11]. However, for $m=0$, the $\sigma$ dependence is observed.

Theorem 1.2. Let $n=3$. Fix $\sigma \in(0, \pi / 2]$. For any $k \in \mathbf{N}$, there exist $(k+1)$ distinct eigenvalues $\lambda_{\varepsilon, k, q, \sigma}(q=0,1, \ldots, k)$ of $-\Lambda$ exist near $k(k+2)$. As $\varepsilon \rightarrow+0$, the following asymptotic expansions hold:

(i) $\sigma \in(0, \pi / 2)$ :

$$
\lambda_{\varepsilon, k, q, \sigma}-k(k+2)=c_{k, q, \sigma, 3} \varepsilon^{\max \{2 q+1,2\}}+o\left(\varepsilon^{\max \{2 q+1,2\}}\right)
$$

with

$$
c_{k, q, \sigma, 3}= \begin{cases}-\frac{2^{2(q+1)}(k+1) q(k+q+1) !(q !)^{2}}{(k-q) !\{2(q+1)\} !(2 q) ! \pi}, & q \geq 1, \\ \frac{2(k+1)^{2} \tan \sigma}{\pi}, & q=0 .\end{cases}
$$

(ii) $\sigma=\pi / 2$ :

$$
\lambda_{\varepsilon, k, q, \sigma}-k(k+2)=c_{k, q, \sigma, 3} \varepsilon^{2 q+1}+o\left(\varepsilon^{2 q+1}\right)
$$


with

$$
c_{k, q, \sigma, 3}=\frac{2^{2(q+1)}(k+1)(q+1)(k+q+1) !(q !)^{2}}{(k-q) !\{2(q+1)\} !(2 q) ! \pi}
$$

Moreover, the multiplicity of $\lambda_{\varepsilon, k, q, \sigma}$ is $(2 q+1)$ if $q \geq 1$ and that of $\lambda_{\varepsilon, k, 0, \sigma}$ is 1 .

Remark 1.2. Similarly to Theorem 1.1 , when $q \geq 1$, the leading term in the right-hand side is not dependent on $\sigma \in[0, \pi / 2)$. When $q=0$, the leading term of eigenvalues depends on $\sigma$. If $q=0$ and $\sigma=0$ (the Neumann condition), the asymptotic order is $\varepsilon^{3}$ by [6].

In Theorems $1.1,1.2$, the case of $k=0$ also holds true for $m=0$ and $q=0$, respectively.

Remark 1.3. Theorems 1.1 and 1.2 show that the domain-monotonicity property (see e.g. pp. 2-5 of $\mathrm{Ni}[15]$ ) does not necessarily hold under the Robin condition, either.

Remark 1.4. It is known by Tichmarsh [22, 23] that there exist no eigenvalues other than those obtained by the separation of variables.

This paper is organized as follows. In Section 2, we review several fundamental properties of the special functions: the Gauss hypergeometric functions, the Gamma function and the psi (di-Gamma) function. In Section 3, we discuss the two dimensional case and in Section 4, we consider the three dimensional case. We use the properties of the associated Legendre functions and the Gauss hypergeometric functions. The analysis of those functions are essential in this paper.

\section{Review of the Gauss hypergeomtric functions and the associated Legendre function}

To investigate the eigenfunctions of the Laplace-Beltrami operator we need to use the spherical harmonic functions and the related special functions. In this section we collect the properties of special functions, the Gauss hypergeometric function $F(a, b, c ; z)$, the Gamma function $\Gamma(z)$ and the psi function $\psi(z)$ used in the successive sections.

Note that all the special functions listed in this section are analytic except for their poles. If the remainder terms of those functions are of $O\left(|z|^{\zeta}\right)$ order, then their derivatives are of $O\left(|z|^{\zeta-1}\right)$.

The definition of the Gauss hypergeomtric function $F(a, b, c ; z)$ is as follows:

$$
F(a, b, c, z)=\frac{\Gamma(c)}{\Gamma(a) \Gamma(b)} \sum_{n=0}^{\infty} \frac{\Gamma(a+n) \Gamma(b+n)}{\Gamma(c+n)} \cdot \frac{z^{n}}{n !}
$$


with $a, b, c \in \mathbf{C} \backslash\{-1,-2,-3, \ldots\}$ and $z$ is a complex variable. If $|z|$ is very small, we have

$$
F(a, b, c, z)=1+\frac{a b}{c} z+O\left(|z|^{2}\right)
$$

For the Gauss hypergeometric functions, the following conversion formula is useful (see pp. 270-274 in Beals and Wong [8]). Let $a, b, c$ be real numbers such that none of $c, a+b+1-c$ and $c+1-a-b$ is a non-positive integer. Then for $x \in(-1,1)$, there holds

$$
\begin{aligned}
& F(a, b, c ; x) \\
& =\frac{\Gamma(c) \Gamma(c-a-b)}{\Gamma(c-a) \Gamma(c-b)} F(a, b, a+b+1-c ; 1-x) \\
& \quad+\frac{\Gamma(c) \Gamma(a+b-c)}{\Gamma(a) \Gamma(b)}(1-x)^{c-a-b} F(c-a, c-b, 1+c-a-b ; 1-x) .
\end{aligned}
$$

The associated Legendre function is defined by

$$
P_{v}^{\mu}(t)=\frac{e^{\mu \pi i}}{\Gamma(1-\mu)}\left(\frac{1+t}{1-t}\right)^{\mu / 2} F\left(-v, v+1,1-\mu ; \frac{1-t}{2}\right) .
$$

in the interval $(-1,1)$. If $\mu$ is a non-negative integer $m$, then it is also expressed by

$$
P_{v}^{m}(t)=\frac{\Gamma(1+v+m)}{m ! 2^{m} \Gamma(1+v-m)}\left(1-t^{2}\right)^{m / 2} F\left(m-v, m+v+1,1+m ; \frac{1-t}{2}\right) .
$$

We recall that this function $P=P_{v}^{m}$ is a solution of the associated Legendre differential equation

$$
\left(1-t^{2}\right) P^{\prime \prime}(t)-2 t P^{\prime}(t)+\left(v(v+1)-\frac{m^{2}}{1-t^{2}}\right) P=0 .
$$

The recursion formula is given by

$$
\left(1-t^{2}\right) \frac{d}{d t} P_{v}^{\mu}(t)=(v+1) t P_{v}^{\mu}(t)-(v-\mu+1) P_{v+1}^{\mu}(t) .
$$

For the Gauss hypergeometric function, there is a conversion formula, which is useful for the analysis on the three dimensional case.

We introduce the psi (or di-Gamma) function $\psi(z)$ defined as

$$
\psi(z)=\frac{\Gamma^{\prime}(z)}{\Gamma(z)} .
$$

The psi function has the following property:

$$
\psi(z+1)=\psi(z)+\frac{1}{z}
$$


The limiting behavior of the Gamma function and the psi function are as follows (see pp. 19-34 in [8] or pp. 2-11 in Moriguchi, Udagawa and Hitotsumatsu $[14])$.

Lemma 2.1. Let $n$ be a positive integer. Then there hold

(i) $\lim _{\tau \rightarrow 0} \tau \Gamma(-n-\tau)=\frac{(-1)^{n+1}}{n !}$.

(ii) $\lim _{\tau \rightarrow 0} \tau \psi(-n-\tau)=1$.

Also, the power series representation of $\psi$ is useful.

LEMMA 2.2. The following expansion holds

$$
\psi(x)=-\gamma-\sum_{n=0}^{\infty}\left(\frac{1}{x+n}-\frac{1}{n+1}\right),
$$

where $\gamma$ is the Euler number defined as

$$
\gamma=\lim _{n \rightarrow \infty}\left(\sum_{m=1}^{n} \frac{1}{m}-\log n\right) .
$$

Let $\alpha, \beta$ be non-integer values and $\ell$ be a positive integer. The function $U(\alpha, \beta, \ell, x)$ is defined as

$$
\begin{aligned}
& U(\alpha, \beta, \ell ; x) \\
&=\frac{(-1)^{\ell}}{\Gamma(\alpha+1-\ell) \Gamma(\beta+1-\ell)(\ell-1) !}[F(\alpha, \beta, \ell ; x) \log x \\
&\left.+\sum_{n=0}^{\infty} \frac{(\alpha)_{n}(\beta)_{n}}{(\ell)_{n} n !}\{\psi(\alpha+n)+\psi(\beta+n)-\psi(n+1)-\psi(\ell+n)\} x^{n}\right] \\
&+\frac{(\ell-2) !}{\Gamma(\alpha) \Gamma(\beta)} x^{1-\ell} \sum_{n=0}^{\ell-2} \frac{(\alpha+1-\ell)_{n}(\beta+1-\ell)_{n}}{(2-\ell)_{n} n !} x^{n}
\end{aligned}
$$

where $(\alpha)_{n}=\alpha(\alpha+1)(\alpha+2) \cdots(\alpha+n-1)=\Gamma(\alpha+n) / \Gamma(n)$ and we agree that the last term in (2.9) is void if $\ell=1$ (see [8, Section 8.4 (pp. 274-276)]). It is known that $U(\alpha, \beta, \ell ; x)$ solves the hypergeometric differential equation

$$
x(1-x) \frac{d^{2} U}{d x^{2}}+\{\ell-(\alpha+\beta+1) x\} \frac{d U}{d x}-\alpha \beta U=0
$$


and is linearly independent of $F(\alpha, \beta, \ell ; x)$, which is also a solution to (2.10). If $\alpha+\beta+1-\ell$ is a non-positive integer,

$$
F(\alpha, \beta, \ell ; x)=\Gamma(\ell) U(\alpha, \beta, \alpha+\beta+1-\ell ; 1-x)
$$

by $[8$, p. 276].

We often use the following formula:

$$
\begin{aligned}
& \Gamma(z+1)=z \Gamma(z), \\
& \Gamma(z) \Gamma(1-z)=\frac{\pi}{\sin \pi z} .
\end{aligned}
$$

\section{In case of $n=2$}

In this section, we treat the $\mathbf{S}^{2}$ case. We use the polar coordinates:

$$
\left\{\begin{array}{l}
x_{1}=\cos \phi \sin \theta \\
x_{2}=\sin \phi \sin \theta \\
x_{3}=\cos \theta
\end{array}\right.
$$

Then $\Omega_{\varepsilon}$ is expressed as

$$
\Omega_{\varepsilon}=\{(\theta, \phi) \mid 0 \leq \theta<\pi-\varepsilon, 0 \leq \phi \leq 2 \pi\}
$$

and the Laplace-Beltrami operator $\Lambda$ yields to

$$
\Lambda v=\frac{\partial^{2} v}{\partial \theta^{2}}+\cot \theta \frac{\partial v}{\partial \theta}+\frac{1}{\sin ^{2} \theta} \frac{\partial^{2} v}{\partial \phi^{2}} .
$$

The eigenvalue problem (1.1) is reduced to

$$
\frac{\partial^{2} v}{\partial \theta^{2}}+\cot \theta \frac{\partial v}{\partial \theta}+\frac{1}{\sin ^{2} \theta} \frac{\partial^{2} v}{\partial \phi^{2}}+\lambda v=0
$$

and (3.1) can be solved by the separation of variables.

Let $v(\theta, \phi)=\Phi(\theta) \Psi(\phi)$. Then we have

$$
\sin ^{2} \theta\left\{\Phi^{\prime \prime}(\theta)+(\cot \theta) \Phi^{\prime}(\theta)+\lambda \Phi\right\}=-\frac{\Psi^{\prime \prime}(\phi)}{\Psi(\phi)}=m^{2}
$$

with $m=0,1,2, \ldots$. Hence we have $\Psi(\phi)=c_{1} \cos m \phi+c_{2} \sin m \phi$. In case of $m=0$, we agree that $\Psi(\phi) \equiv 1$. Also, $\Phi$ satisfies

$$
\Phi^{\prime \prime}(\theta)+(\cot \theta) \Phi^{\prime}(\theta)+\left(\lambda-\frac{m^{2}}{\sin ^{2} \theta}\right) \Phi=0 .
$$

By letting $t=\cos \theta$ and $\Phi(\theta)=P(t)$, we have

$$
\left(1-t^{2}\right) P^{\prime \prime}(t)-2 t P^{\prime}(t)+\left(\lambda-\frac{m^{2}}{1-t^{2}}\right) P=0 .
$$


As seen in Section 2, the associated Legendre function $P_{v}^{m}(\cos \theta)$ is the regular solution of (3.2) where $\lambda=v(v+1)$ with $v>0$. The constant $v$ is determined by the boundary condition

$$
\left.\cos \sigma \frac{d}{d \theta} P_{v}^{m}(\cos \theta)\right|_{\theta=\pi-\varepsilon}+\sin \sigma P_{v}^{m}(\cos (\pi-\varepsilon))=0 .
$$

Using the recursion formula (2.7), we have

$$
\frac{d}{d \theta} P_{v}^{\mu}(\cos \theta)=\frac{d}{d t} P_{v}^{\mu}(t)\left(-\sqrt{1-t^{2}}\right)=-\frac{(v+1) t P_{v}^{\mu}(t)-(v-\mu+1) P_{v+1}^{\mu}(t)}{\sqrt{1-t^{2}}} .
$$

Thus the Robin boundary condition can be rewritten as

$$
\begin{aligned}
& \{(v+1) \cos \sigma \cos (\pi-\varepsilon)-\sin \sigma \sin \varepsilon\} P_{v}^{m}(\cos (\pi-\varepsilon)) \\
& =(v-m+1) \cos \sigma P_{v+1}^{m}(\cos (\pi-\varepsilon)) .
\end{aligned}
$$

First we show the existence of an eigenvalue near $k(k+1)$ to (3.2) under the condition

$$
\cos \sigma \Phi^{\prime}(\pi-\varepsilon)+\sin \sigma \Phi(\pi-\varepsilon)=0
$$

Note that (3.2) is equavalent to

$$
\left\{(\sin \theta) \Phi^{\prime}\right\}^{\prime}+\lambda(\sin \theta) \Phi-\frac{m^{2}}{\sin \theta} \Phi=0 .
$$

Lemma 3.1. For each $k \in \mathbf{N}$, there exists a unique eigenvalue $\lambda_{\varepsilon, k, m, \sigma}$ to (3.6) with (3.5) near $k(k+1)$ for any small $\varepsilon>0$.

Proof. We use the Prüfer transform. Let

$$
\left\{\begin{array}{l}
\Phi=\rho(\theta) \cos s(\theta), \\
-(\sin \theta) \Phi^{\prime}=\rho(\theta) \sin s(\theta) .
\end{array}\right.
$$

Substituting (3.7) for (3.6), we have

$$
-\rho^{\prime} \sin s-\rho(\cos s) s^{\prime}+\lambda \rho \cos s-\frac{m^{2}}{\sin \theta} \rho \cos s=0
$$

and

$$
\rho^{\prime} \cos s-\rho(\sin s) s^{\prime}=-\frac{\rho \sin s}{\sin \theta} .
$$

Multiplying (3.8) by $\cos s$ and (3.9) by $\sin s$, summing them up, we obtain

$$
\frac{d s}{d \theta}=\lambda \sin \theta \cos ^{2} s+\frac{\sin ^{2} s}{\sin \theta}-\frac{m^{2}}{\sin \theta} \cos ^{2} s .
$$


We take $s(0)=0$ and $\rho(0)=0$ when $m \geq 1$ if otherwise, (3.6) cannot be solvable due to the singularity. While $m=0$, we take $s(0)=0$ and $\rho(0)=1$. Thus, to satisfy the boundary condition, $s(\theta)$ must satisfy

$$
\cos \sigma \sin s(\pi-\varepsilon)=\sin \varepsilon \sin \sigma \cos s(\pi-\varepsilon) .
$$

By the Prüfer comparison theorem [20] (or see Theorem 3 in Section 41 of Yosida [24]; the Prüfer theorem is applicable to (3.6)), it is well known that $s(\theta)$ is monotone increasing with respect to $\lambda$ for any fixed $\theta \in[0, \pi)$, that $\lim _{\lambda \rightarrow \infty} s(\theta)=\infty$ and that $s(0) \leq 0$ by (3.10). Note that $k(k+1)$ is an eigenvalue of $-\Lambda$ on $\mathbf{S}^{2}$, that is, $\sin s(\pi)=0$ since on the whole sphere, $\Phi$ must satisfy $\Phi^{\prime}(\pi)=0$ (otherwise, it cannot be an eigenfunction). By the continuity of solutions to (3.10) with respect to $\theta, \sin s(\pi-\varepsilon)$ is also close to 0 . If $\varepsilon>0$ is sufficiently small, then by the continuity of solutions with respect to the parameter $\lambda$, and the monotonicity with respect to $\lambda$, by (3.11), there must exist unique $\lambda_{\varepsilon, k, m, \sigma}$ near $k(k+1)$.

Proof of Theorem 1.1. The existence of eigenvalues is assured by Lemma 3.1. We prove the asymptotic behavior of the eigenvalue $\lambda=\lambda_{\varepsilon, k, m, \sigma}=$ $v(\varepsilon)(v(\varepsilon)+1)$ near $k(k+1)$ for $k \in \mathbf{N}$, the condition for $m$ and the multiplicity of eigenvalues.

We use the expression of $P_{v}^{m}(t)$ as (2.5). Since $1+m-(m-v)-$ $(m+v+1)=-m<0, P_{v}^{m}(t)$ is singular at $t=-1$ as in the following proposition:

Proposition 3.1 (cf. [14]). Let $\alpha, \beta, \gamma$ be real numbers such that $\alpha+\beta-\gamma$ $>0$ and let $k$ be the greatest integer which does not exceed $\alpha+\beta-\gamma$. Then there holds

$$
\begin{aligned}
\lim _{x \rightarrow 1-0}(F(\alpha, \beta, \gamma ; x) & \\
& \left.-\sum_{n=0}^{k}(-1)^{n} \frac{\Gamma(\alpha+\beta-\gamma-n) \Gamma(\gamma)}{\Gamma(\alpha) \Gamma(\beta) n !}(\gamma-\alpha)_{n}(\gamma-\beta)_{n}(1-x)^{n+\gamma-\alpha-\beta}\right) \\
= & \frac{\Gamma(\gamma) \Gamma(\gamma-\alpha-\beta)}{\Gamma(\gamma-\alpha) \Gamma(\gamma-\beta)}
\end{aligned}
$$

However, in our case, $\alpha=m-v, \beta=m+v+1$ and $\gamma=m+1$, which yield $\gamma-\alpha-\beta=-m$. Hence, $\Gamma(\gamma-\alpha-\beta)$ in the right-hand side is not defined (its absolute value is infinity). Thus, to analyze the eigenvalue problem beyond this difficulty we use $U(\alpha, \beta, \ell, x)$. In our case, $\alpha=m-v, \beta=m+v+1, \ell=m+1$ and $x=\left(1-t_{\varepsilon}\right) / 2$ with $t_{\varepsilon}=\cos (\pi-\varepsilon)$. Thus, (2.5) can be written as

$$
P_{v}^{m}\left(t_{\varepsilon}\right)=\frac{\Gamma(1+v+m) \Gamma(m+1)}{m ! 2^{m} \Gamma(1+v-m)}\left(1-t_{\varepsilon}^{2}\right)^{m / 2} U\left(m-v, m+v+1, m+1 ; \frac{t_{\varepsilon}+1}{2}\right) .
$$

Then the boundary condition (3.4) yields 


$$
\begin{aligned}
-\{(v+1) \cos \sigma \cos \varepsilon+\sin \sigma \sin \varepsilon\} & \frac{\Gamma(1+v+m)}{\Gamma(1+v-m)} \\
& \times U\left(m-v, m+v+1, m+1 ; \frac{t_{\varepsilon}+1}{2}\right) \\
= & (v-m+1) \cos \sigma \frac{\Gamma(2+v+m)}{\Gamma(2+v-m)} U\left(m-v-1, m+v+2, m+1 ; \frac{t_{\varepsilon}+1}{2}\right)
\end{aligned}
$$

and after some elementary calculation, we have

$$
\begin{gathered}
-\{(v+1) \cos \sigma \cos \varepsilon+\sin \sigma \sin \varepsilon\} U\left(m-v, m+v+1, m+1 ; \frac{t_{\varepsilon}+1}{2}\right) \\
=(v+m+1) \cos \sigma U\left(m-v-1, m+v+2, m+1 ; \frac{t_{\varepsilon}+1}{2}\right) .
\end{gathered}
$$

Substituting $\alpha=m-v, \beta=m+v+1$ and $\ell=m+1$ for (2.9), we get

$$
\begin{aligned}
U(m- & v, m+v+1, m+1 ; x) \\
= & \frac{(-1)^{m+1}}{\Gamma(-v) \Gamma(v+1) m !}[F(m-v, m+v+1, m+1 ; x) \log x \\
& +\sum_{n=0}^{\infty} \frac{(m-v)_{n}(m+v+1)_{n}}{(m+1)_{n} n !} \times\{\psi(m-v+n) \\
& \left.+\psi(m+v+1+n)-\psi(n+1)-\psi(m+1+n)\} x^{n}\right] \\
& +\frac{(m-1) !}{\Gamma(m-v) \Gamma(m+v+1)} x^{-m} \sum_{n=0}^{m-1} \frac{(-v)_{n}(v+1)_{n}}{(1-m)_{n} n !} x^{n}
\end{aligned}
$$

and

$$
\begin{aligned}
U(m- & v-1, m+v+2, m+1 ; x) \\
= & \frac{(-1)^{m+1}}{\Gamma(-v-1) \Gamma(v+2) m !}[F(m-v-1, m+v+2, m+1 ; x) \log x \\
& +\sum_{n=0}^{\infty} \frac{(m-v-1)_{n}(m+v+2)_{n}}{(m+1)_{n} n !} \times\{\psi(m-v-1+n) \\
& \left.+\psi(m+v+2+n)-\psi(n+1)-\psi(m+1+n)\} x^{n}\right] \\
& +\frac{(m-1) !}{\Gamma(m-v-1) \Gamma(m+v+2)} x^{-m} \sum_{n=0}^{m-1} \frac{(-v-1)_{n}(v+2)_{n}}{(1-m)_{n} n !} x^{n} .
\end{aligned}
$$

Recall that the last terms in (3.13) and (3.14) are void if $m=0$. 
Let us consider the case where $m$ is a positive integer. Substituting $x=$ $\left(t_{\varepsilon}+1\right) / 2=\varepsilon^{2} / 4+O\left(\varepsilon^{4}\right)$, we have

$$
F\left(m-v, m+v+1, m+1 ; \frac{t_{\varepsilon}+1}{2}\right)=1+c_{1} \varepsilon^{2}+O\left(\varepsilon^{4}\right) .
$$

Thus the first terms in (3.13) and (3.14) are not dominant as $\varepsilon \rightarrow+0$. Set $v=$ $v(\varepsilon)$ and consider the value of $v(0)$ as $\varepsilon \rightarrow+0$. If $v(0)$ is not an integer, then we reach a contradiction. Indeed, if $v(0)$ is not an integer, then the last terms in (3.13) and (3.14) are dominant and $U\left(m-v, m+v+1, m+1,\left(t_{\varepsilon}+1\right) / 2\right)$ and $U\left(m-v-1, m+v+2, m+1,\left(t_{\varepsilon}+1\right) / 2\right)$ are of the same order $\varepsilon^{-2 m}$ as $\varepsilon \rightarrow+0$ according to (3.12). Thus the leading terms of the each side must satisfy

$$
-\frac{(v+1)(m-1) !}{\Gamma(m-v) \Gamma(v+m+1)}\left(\frac{\varepsilon}{2}\right)^{-2 m}=\frac{(v+m+1)(m-1) !}{\Gamma(m-v-1) \Gamma(m+v+2)}\left(\frac{\varepsilon}{2}\right)^{-2 m} .
$$

This leads to $v+1=v-m+1$, which contradicts the assumption $m \neq 0$.

If $m>k$, then (3.16) also implies the contradiction. Thus $m=1,2, \ldots, k$. Summarizing the above argument, we can expect that

$$
v=v(\varepsilon)=k+v^{*}(\varepsilon), \quad v^{*}(\varepsilon)=O\left(\varepsilon^{2 m}\right), \quad m=1,2, \ldots, k,
$$

where $v^{*}(\varepsilon)$ may be of smaller order than that of $\varepsilon^{2 m}$.

In the next step we determine the constant $\lim _{\varepsilon \rightarrow+0} \varepsilon^{-2 m} v^{*}(\varepsilon)$. Among the terms in the parenthesis [ ] of (3.13) and (3.14), we need to find a term which becomes large as $\varepsilon \rightarrow+0$. Lemma 2.1 implies that $\Gamma(z)$ has a pole of order 1 when $z$ is a negative integer. Thus, $\Gamma(m-v), \Gamma(m-v-1)$ and $\Gamma(-v)$ become large as $\varepsilon \rightarrow+0$. At the same time, we note that the function $\psi(z)$ also becomes large as $z$ converges to a negative integer.

First, the term

$$
F\left(m-v, m+v+1, m+1 ; \frac{t_{\varepsilon}+1}{2}\right) \log \frac{t_{\varepsilon}+1}{2}
$$

in (3.13) is neglected since the coefficient of this term has $\Gamma(-v)$ in the denominator if $m \geq 1$. In (3.13),

$$
\sum_{n=0}^{\infty} \frac{(m-v)_{n}(m+v+1)_{n}}{(m+1)_{n} n !} \psi(m-v+n)\left(\frac{t_{\varepsilon}+1}{2}\right)^{n}
$$

can be large as $v \rightarrow k$. Since $\left\{\left(t_{\varepsilon}+1\right) / 2\right\}^{n}=(\varepsilon / 2)^{2 n}+O\left(\varepsilon^{2 n+2}\right)$, then the first term in the sum is the leading term. By Lemma 2.1, we note that

$$
\lim _{v \rightarrow k} \frac{\psi(m-v)}{\Gamma(-v)}=(-1)^{k+1} k !
$$

The same arguments in this paragraph apply to (3.14). Then, finally, we see that (3.12) yields 
$-\{(v+1) \cos \sigma \cos \varepsilon+\sin \sigma \sin \varepsilon\}$

$$
\begin{aligned}
& \times\left[\frac{(-1)^{m+1} \psi(m-v)}{\Gamma(-v) \Gamma(v+1) m !}+\frac{(m-1) !}{\Gamma(m-v) \Gamma(m+v+1)}\left\{\left(\frac{\varepsilon}{2}\right)^{-2 m}+O\left(\varepsilon^{-2 m+2}\right)\right\}\right] \\
& =(v+m+1) \cos \sigma \\
& \times\left[\frac{(-1)^{m+1} \psi(m-v-1)}{\Gamma(-v-1) \Gamma(v+2) m !}+\frac{(m-1) !}{\Gamma(m-v-1) \Gamma(m+v+2)}\right. \\
& \left.\quad \times\left\{\left(\frac{\varepsilon}{2}\right)^{-2 m}+O\left(\varepsilon^{-2 m+2}\right)\right\}\right] .
\end{aligned}
$$

As $\varepsilon \rightarrow+0$, there holds

$$
\begin{aligned}
& -\left(\cos \sigma \cos \varepsilon+\frac{\sin \sigma}{v+1} \sin \varepsilon\right) \\
& \quad \times\left\{\frac{(-1)^{m+k+2} k !}{k ! m !}+\frac{(-1)^{k-m+1}(m-1) !(k-m) !}{(m+k) !} 4^{m} \tilde{c}_{k, m, \sigma, 2}(\varepsilon)+O(\varepsilon)\right\} \\
& =\frac{v+m+1}{v+1} \cos \sigma \\
& \quad \times\left\{\frac{(-1)^{m+k+3}(k+1) !}{(k+1) ! m !}+\frac{(-1)^{m-k+2}(m-1) !(k+1-m) !}{(m+k+1) !} 4^{m} \tilde{c}_{k, m, \sigma, 2}(\varepsilon)+O(\varepsilon)\right\},
\end{aligned}
$$

where $v^{*}(\varepsilon)=\tilde{c}_{k, m, \sigma, 2}(\varepsilon) \varepsilon^{2 m}+O\left(\varepsilon^{2 m+1}\right)$. Then we see that

$$
\tilde{c}_{k, m, \sigma, 2}(\varepsilon)=\frac{-\frac{m}{k+1} \cos \sigma+\frac{\sin \varepsilon}{k+1} \sin \sigma}{\frac{m}{k+1} \cos \sigma+\frac{\sin \varepsilon}{k+1} \sin \sigma} \frac{(m+k) !}{4^{m}(k-m) ! m !(m-1) !}+O(\varepsilon) .
$$

Taking the limit as $\varepsilon \rightarrow+0$ in (3.17), we conclude that the limit $\lim _{\varepsilon \rightarrow+0} \tilde{c}_{k, m, \sigma, 2}(\varepsilon)$ exists as

$$
\tilde{c}_{k, m, \sigma, 2}:=\lim _{\varepsilon \rightarrow+0} \tilde{c}_{k, m, \sigma, 2}(\varepsilon)=-\frac{(m+k) !}{4^{m}(k-m) ! m !(m-1) !}
$$

if $\sigma \in[0, \pi / 2)$ and

$$
\tilde{c}_{k, m, \pi / 2,2}:=\lim _{\varepsilon \rightarrow+0} \tilde{c}_{k, m, \pi / 2,2}(\varepsilon)=\frac{(m+k) !}{4^{m}(k-m) ! m !(m-1) !},
$$

if $\sigma=\pi / 2$, which coincides with a result in [11]. 
Next we consider the case $m=0$. In this case, the situation is different from the case $m \geq 1$. In (3.13), $\log (\cos (\pi-\varepsilon)+1) / 2$ may not be neglected. Although $\psi(v+1), \psi(v+2)$ are uniformly bounded, they cannot be neglected and so cannot be $\psi(1)$ either.

Indeed, as $v \rightarrow k$, the leading terms are as follows and they must satisfy

$$
\begin{aligned}
&-\frac{\cos \sigma \cos \varepsilon+\frac{\sin \sigma}{k+1} \sin \varepsilon}{\Gamma(-v) \Gamma(v+1)}\{ F\left(-v, v+1,1 ; \frac{t_{\varepsilon}+1}{2}\right) \log \frac{t_{\varepsilon}+1}{2} \\
&+\left.\psi(-v)+\psi(v+1)-2 \psi(1)+O\left(\varepsilon^{2}\right)\right\} \\
&=-\frac{\cos \sigma}{\Gamma(-v-1) \Gamma(v+2)}\{ F\left(-v-1, v+2,1 ; \frac{t_{\varepsilon}+1}{2}\right) \log \frac{t_{\varepsilon}+1}{2} \\
&\left.+\psi(-v-1)+\psi(v+2)-2 \psi(1)+O\left(\varepsilon^{2}\right)\right\}
\end{aligned}
$$

with $t_{\varepsilon}=\cos (\pi-\varepsilon)=-1+\varepsilon^{2} / 2+O\left(\varepsilon^{4}\right)$. In this case, we need to determine $c_{1}$ in (3.15) exactly and there hold

$$
F\left(-v, v+1,1 ; \frac{t_{\varepsilon}+1}{2}\right)=1-\frac{v(v+1)}{4} \varepsilon^{2}+O\left(\varepsilon^{4}\right)
$$

and

$$
F\left(-v-1, v+2,1 ; \frac{t_{\varepsilon}+1}{2}\right)=1-\frac{(v+1)(v+2)}{4} \varepsilon^{2}+O\left(\varepsilon^{4}\right) .
$$

Thus, we see

$$
\begin{aligned}
& -\left(\cos \sigma+\frac{\sin \sigma}{v+1} \varepsilon+O\left(\varepsilon^{2}\right)\right) \\
& \times\left[\left\{1-\frac{v(v+1)}{4} \varepsilon^{2}\right\} \log \left(\frac{\varepsilon}{2}\right)^{2}\right. \\
& \left.+\psi(-v)+\psi(v+1)-2 \psi(1)+O\left(\varepsilon^{2}\right)\right] \\
& =-\cos \sigma\left[\left\{1-\frac{(v+1)(v+2)}{4} \varepsilon^{2}\right\} \log \left(\frac{\varepsilon}{2}\right)^{2}\right. \\
& \left.\quad+\psi(-v-1)+\psi(v+2)-2 \psi(1)+O\left(\varepsilon^{2}\right)\right]
\end{aligned}
$$

which implies 


$$
\begin{aligned}
-\left(\frac{\sin \sigma}{v+1} \varepsilon+O\left(\varepsilon^{2}\right)\right) \\
\quad \times\left[\left\{1-\frac{v(v+1)}{4} \varepsilon^{2}\right\} \log \left(\frac{\varepsilon}{2}\right)^{2}+\psi(-v)+\psi(v+1)-2 \psi(1)+O\left(\varepsilon^{2}\right)\right] \\
=\left\{\frac{(v+1)(v+2)}{2} \varepsilon^{2} \log \left(\frac{\varepsilon}{2}\right)^{2}-\psi(-v-1)+\psi(-v)\right. \\
\left.\quad-\psi(v+2)+\psi(v+1)+O\left(\varepsilon^{2}\right)\right\} \cos \sigma .
\end{aligned}
$$

Using

$$
\psi(-v)=\psi(-v-1)-\frac{1}{v+1}, \quad \psi(v+2)=\psi(v+1)+\frac{1}{v+1},
$$

we obtain

$$
\begin{aligned}
-\left(\frac{\sin \sigma}{v+1} \varepsilon+O\left(\varepsilon^{2}\right)\right) \\
\times\left[\left\{1-\frac{v(v+1)}{4} \varepsilon^{2}\right\} \log \left(\frac{\varepsilon}{2}\right)^{2}+\psi(-v)\right. \\
\left.+\psi(v+1)-2 \psi(1)+O\left(\varepsilon^{2}\right)\right] \\
=-\frac{2 \cos \sigma}{v+1}+\frac{(v+1)(v+2) \cos \sigma}{2} \varepsilon^{2} \log \left(\frac{\varepsilon}{2}\right)^{2}+O\left(\varepsilon^{2}\right) .
\end{aligned}
$$

Thus, we see that

$$
-\frac{\varepsilon \sin \sigma}{v+1} \psi(-v)=-\frac{2 \cos \sigma}{v+1}+o(1)
$$

As before, since $\psi(z)$ has a pole of order one at a negative integer and there exists a precise expansion of $\psi$ (see Lemma 2.2). Substituting $v=k+v^{*}(\varepsilon)$ in Lemma 2.2 as $\varepsilon \rightarrow+0$, we see that $\psi\left(-k-v^{*}(\varepsilon)\right) \sim 1 / v^{*}(\varepsilon)$. If $\sigma \in(0, \pi / 2)$, we obtain

$$
\lim _{\varepsilon \rightarrow 0} \frac{v^{*}(\varepsilon)}{\varepsilon}=\frac{\tan \sigma}{2}=\tilde{c}_{k, 0, \sigma, 2}
$$

We remark the behavior for the Dirichlet boundary condition $\sigma=\pi / 2$. From (3.20), we have

$$
\log \left(\frac{\varepsilon}{2}\right)^{2}+\psi(-v)=O(1)
$$


Then we have

$$
v^{*}(\varepsilon)=\frac{1}{-2 \log \varepsilon}(1+o(1)),
$$

which is the same result as $[11,13]$.

We conclude that $v=v(\varepsilon)=k+\tilde{c}_{k, m, \sigma, 2} \varepsilon^{2 m}+o\left(\varepsilon^{2 m}\right)$ for some constant $\tilde{c}_{k, m, \sigma, 2}$ and that for each $m \in[1, k]$,

$$
P_{v(k, m, \varepsilon)}^{m}(\cos \theta)\left(c_{1} \cos m \phi+c_{2} \sin m \phi\right)
$$

are a pair of the eigenfunctions corresponding to the eigenvalue

$$
\lambda=v(\varepsilon)(v(\varepsilon)+1)=k(k+1)+(2 k+1) \tilde{c}_{k, m, \sigma, 2} \varepsilon^{2 m}+O\left(\varepsilon^{2 m+2}\right)
$$

with $m=1,2,3, \ldots, k$.

In case of $m=0, P_{v(k, 0, \varepsilon)}^{0}(\cos \theta)$ is the eigenfunction corresponding to

$$
\lambda=v(\varepsilon)(v(\varepsilon)+1)=k(k+1)+(2 k+1) \tilde{c}_{k, 0, \sigma, 2} \varepsilon+o(\varepsilon) .
$$

The multiplicity of eigenvalues is obvious from the expressions above. Thus, we take $c_{k, m, \sigma, 2}=(2 k+1) \tilde{c}_{k, m, \sigma, 2}$ and the conclusion follows.

\section{In case of $n=3$}

As in Section 3, we also use the polar coordinates:

$$
\left\{\begin{array}{l}
x_{1}=\cos \phi \sin \varphi \sin \theta \\
x_{2}=\sin \phi \sin \varphi \sin \theta \\
x_{3}=\cos \varphi \sin \theta \\
x_{4}=\cos \theta .
\end{array}\right.
$$

Here the domain $\Omega_{\varepsilon}$ is expressed as

$$
\Omega_{\varepsilon}=\{(\theta, \varphi, \phi) \mid 0 \leq \theta<\pi-\varepsilon, 0 \leq \varphi \leq \pi, 0 \leq \phi \leq 2 \pi\}
$$

and so is $\Lambda v$ as

$$
\Lambda v=\frac{1}{\sin ^{2} \theta} \frac{\partial}{\partial \theta}\left(\sin ^{2} \theta \frac{\partial v}{\partial \theta}\right)+\frac{1}{\sin ^{2} \theta \sin \varphi} \frac{\partial}{\partial \varphi}\left(\sin \varphi \frac{\partial v}{\partial \varphi}\right)+\frac{1}{\sin ^{2} \theta \sin ^{2} \varphi} \frac{\partial^{2} v}{\partial \phi^{2}} .
$$

Then separating variables

$$
v(\theta, \varphi, \phi)=U(\theta) V(\varphi) W(\phi),
$$

we get

$$
\left\{\begin{array}{l}
\frac{1}{U} \frac{\partial}{\partial \theta}\left(\sin ^{2} \theta \frac{\partial U}{\partial \theta}\right)+\lambda \sin ^{2} \theta=\ell \\
-\frac{1}{V \sin \varphi} \frac{\partial}{\partial \varphi}\left(\sin \varphi \frac{\partial V}{\partial \varphi}\right)-\frac{1}{W \sin ^{2} \varphi} \frac{\partial^{2} W}{\partial \phi^{2}}=\ell
\end{array}\right.
$$


for some constant $\ell$. Again, the second equation of (4.1) is reduced to

$$
\left\{\begin{array}{l}
\sin \varphi \frac{\partial}{\partial \varphi}\left(\sin \varphi \frac{\partial V}{\partial \varphi}\right)+\ell\left(\sin ^{2} \varphi\right) V=L V, \\
-\frac{\partial^{2} W}{\partial \phi^{2}}=L W
\end{array}\right.
$$

for some constant $L$. Since $W$ is periodic, $L=m^{2}$ with $m=0,1,2, \ldots$ and thus

$$
W=c_{1} \cos m \phi+c_{2} \sin m \phi .
$$

We set $W \equiv 1$ when $m=0$. Then $V$ satisfies

$$
V^{\prime \prime}(\varphi)+(\cot \varphi) V^{\prime}(\varphi)+\left(\ell-\frac{m^{2}}{\sin ^{2} \varphi}\right) V=0 .
$$

Put $t=\cos \varphi$ and $P(t)=V(\varphi)$. It is a solution of the associated Legendre differential equation (2.6). Let $\ell=\hat{v}(\hat{v}+1)$. Then its solution is expressed as $P(t)=P_{\hat{v}}^{m}(t)$. Since the solutions are regular at $t=1, \hat{v}$ is a positive integer $q$ and

$$
q \geq m \text {. }
$$

By (4.1) with $\ell=q(q+1), U$ satisfies

$$
U^{\prime \prime}(\theta)+2(\cot \theta) U^{\prime}(\theta)+\left\{\lambda-\frac{q(q+1)}{\sin ^{2} \theta}\right\} U=0,
$$

which is called the "hyper-sphere differential equation". As in Section 3, (4.5) is equivalent to

$$
\left\{\left(\sin ^{2} \theta\right) \Phi^{\prime}\right\}^{\prime}+\lambda\left(\sin ^{2} \theta\right) \Phi-q(q+1) \Phi=0 .
$$

Let $t=\cos \theta$ and $U(\theta)=\tilde{U}(t) /\left(1-t^{2}\right)^{1 / 4}$. Then $\tilde{U}(t)$ satisfies the associated Legendre differential equation

$$
\left(1-t^{2}\right) \tilde{U}^{\prime \prime}(t)-2 t \tilde{U}^{\prime}(t)+\left\{\lambda+\frac{3}{4}-\frac{q(q+1)+1 / 4}{1-t^{2}}\right\} \tilde{U}(t)=0
$$

We see that $\tilde{U}(t)$ is expressed as $\tilde{U}(t)=P_{v}^{\alpha}(t)$ with

$$
v(v+1)=\lambda+\frac{3}{4}, \quad \alpha^{2}=q(q+1)+\frac{1}{4}=\left(q+\frac{1}{2}\right)^{2} .
$$

Then $U$ can be written as

$$
U(\theta)=\frac{P_{v}^{-(q+1 / 2)}(\cos \theta)}{\sqrt{\sin \theta}} .
$$

Thus the eigenfunctions of (1.1) are of the form

$$
\Phi=\frac{P_{v}^{-(q+1 / 2)}(\cos \theta)}{\sqrt{\sin \theta}} P_{q}^{m}(\cos \varphi)\left(c_{1} \cos m \phi+c_{2} \sin m \phi\right) .
$$


Similarly to (3.4), we determine $v=v(\varepsilon)$ as a function of $\varepsilon$ so that the Robin boundary condition is satisfied, i.e.

$$
\left.\cos \sigma \frac{d}{d \theta} U(\theta)\right|_{\theta=\pi-\varepsilon}+\sin \sigma U(\pi-\varepsilon)=0
$$

Consequently, we have

$$
\begin{aligned}
-\left.\cos \sigma \sin ^{2}(\pi-\varepsilon) \frac{d}{d t} P_{v}^{-(q+1 / 2)}(t)\right|_{t=\cos (\pi-\varepsilon)} & \\
& -\frac{\cos \sigma}{2} P_{v}^{-(q+1 / 2)}(\cos (\pi-\varepsilon)) \cos (\pi-\varepsilon) \\
& +\sin \sigma \sin (\pi-\varepsilon) P_{v}^{-(q+1 / 2)}(\cos (\pi-\varepsilon)) \sin (\pi-\varepsilon)=0 .
\end{aligned}
$$

From this relation and the recursion formula (2.7) with $\mu=-(q+1 / 2)$, we get

$$
\begin{gathered}
\left\{\left(v+\frac{3}{2}\right) \cos \sigma \cos (\pi-\varepsilon)-\sin \sigma \sin (\pi-\varepsilon)\right\} P_{v}^{-(q+1 / 2)}(\cos (\pi-\varepsilon)) \\
=\left(v+q+\frac{3}{2}\right) \cos \sigma P_{v+1}^{-(q+1 / 2)}(\cos (\pi-\varepsilon)) .
\end{gathered}
$$

We express $P_{v}^{\mu}$ in terms of the Gauss hypergeometric functions as

$$
\begin{aligned}
e^{(q+1 / 2) \pi i} P_{v}^{-(q+1 / 2)}(t) & \\
\quad= & \frac{1}{\Gamma(q+3 / 2)}\left(\frac{1+t}{1-t}\right)^{-(q+1 / 2) / 2} F\left(-v, v+1, q+\frac{3}{2} ; \frac{1-t}{2}\right),
\end{aligned}
$$

using (2.4) with $\mu=-(q+1 / 2)$. As in Section 3, we first show the existence of eigenvalues. Parallel to Lemma 3.1, we have the following.

Lemma 4.1. For each $k \in \mathbf{N}$, there exists a unique eigenvalue $\lambda_{\varepsilon, k, m, \sigma}$ to (4.6) with (3.5) near $k(k+2)$ for any small $\varepsilon>0$.

Proof. As in the proof of Lemma 3.1, we use the Prüfer transform

$$
\left\{\begin{array}{l}
\Phi=\rho(\theta) \cos s(\theta), \\
-\left(\sin ^{2} \theta\right) \Phi^{\prime}=\rho(\theta) \sin s(\theta) .
\end{array}\right.
$$

Substituting (4.11) for (4.6), we have

$$
-\rho^{\prime} \sin s-\rho(\cos s) s^{\prime}+\lambda \rho \sin ^{2} s \cos s-q(q+1) \rho \cos s=0
$$

and

$$
\rho^{\prime} \cos s-\rho(\sin s) s^{\prime}=-\frac{\rho \sin s}{\sin ^{2} \theta} .
$$


Multiplying (4.12) by $\cos s$ and (4.13) by $\sin s$, we obtain

$$
\frac{d s}{d \theta}=\lambda \sin ^{2} \theta \cos ^{2} s(\theta)+\frac{\sin ^{2} s(\theta)}{\sin ^{2} \theta}-q(q+1) \cos ^{2} s(\theta) .
$$

The boundary condition yields

$$
\cos \sigma \sin s(\pi-\varepsilon)=\sin \sigma \sin ^{2}(\pi-\varepsilon) \cos s(\pi-\varepsilon) .
$$

The Prüfer comparison theorem is applicable to (4.14) and the rest part of proof is proved as in the proof of Lemma 3.1.

Proof of Theorem 1.2. As in the proof of Theorem 1.1, the existence of eigenvalues is ensured by Lemma 4.1. We consider the asymptotic behavior. From (4.9) and (4.10) we deduce that

$$
\begin{gathered}
\left\{-\left(v+\frac{3}{2}\right) \cos \sigma \cos \varepsilon-\sin \sigma \sin \varepsilon\right\} F\left(-v, v+1, q+\frac{3}{2} ; \frac{1-t_{\varepsilon}}{2}\right) \\
=\left(v+q+\frac{3}{2}\right) \cos \sigma F\left(-(v+1), v+2, q+\frac{3}{2} ; \frac{1-t_{\varepsilon}}{2}\right) .
\end{gathered}
$$

Since the hypergeometric functions in the above equation are singular at $t=-1$, we use the following identity (see (2.3) in Section 2)

$$
\begin{aligned}
F\left(-v, v+1, q+\frac{3}{2} ; \frac{1-t_{\varepsilon}}{2}\right) \\
=\frac{\Gamma\left(q+\frac{3}{2}\right) \Gamma\left(q+\frac{1}{2}\right)}{\Gamma\left(q+v+\frac{3}{2}\right) \Gamma\left(q-v+\frac{1}{2}\right)} F\left(-v, v+1,-q+\frac{1}{2} ; \frac{t_{\varepsilon}+1}{2}\right) \\
\quad+\frac{\Gamma\left(q+\frac{3}{2}\right) \Gamma\left(-q-\frac{1}{2}\right)}{\Gamma(-v) \Gamma(v+1)}\left(\frac{t_{\varepsilon}+1}{2}\right)^{q+1 / 2} \\
\quad \times F\left(q+v+\frac{3}{2}, q-v+\frac{1}{2}, q+\frac{3}{2} ; \frac{t_{\varepsilon}+1}{2}\right) .
\end{aligned}
$$

Assume that $v(\varepsilon)=v(0)+o(1)$ and let us take the limit as $\varepsilon \rightarrow+0$ in (4.16). Since $F\left(-v(0), v(0)+1,-q+\frac{1}{2} ; 0\right)=1, F\left(q+v(0)+\frac{3}{2}, q-v(0)+\frac{1}{2}, q+\frac{3}{2} ; 0\right)=1$ and $q+3 / 2-\{-v+(v+1)\}=q+1 / 2>0$, we find

$$
F(-v(0), v(0)+1, q+3 / 2 ; 1)=\frac{\Gamma\left(q+\frac{3}{2}\right) \Gamma\left(q+\frac{1}{2}\right)}{\Gamma(q+v(0)+3 / 2) \Gamma(q-v(0)+1 / 2)} .
$$

If $q-v(0)+1 / 2$ is not a negative integer, then we will have a contradiction. Indeed, suppose that $q-v(0)+1 / 2$ is not a negative integer. The second term in (4.16) vanishes as $\varepsilon \rightarrow+0$ and (4.16) leads to 


$$
\begin{aligned}
& -\left(v(0)+\frac{3}{2}\right) \frac{\Gamma\left(q+\frac{3}{2}\right) \Gamma\left(q+\frac{1}{2}\right)}{\Gamma\left(q+v(0)+\frac{3}{2}\right) \Gamma\left(q-v(0)+\frac{1}{2}\right)} \\
& =\left(v(0)+q+\frac{3}{2}\right) \frac{\Gamma\left(q+\frac{3}{2}\right) \Gamma\left(q+\frac{1}{2}\right)}{\Gamma\left(q+v(0)+\frac{5}{2}\right) \Gamma\left(q-v(0)-\frac{1}{2}\right)} .
\end{aligned}
$$

for $\varepsilon=0$. The formula (2.12) implies

$$
v(0)+\frac{3}{2}=v(0)+\frac{1}{2}-q
$$

provided $\Gamma(q-v(0)+1 / 2) \neq \infty$ and thus $q=-1$, which contradicts (4.4). Thus the only possibility for the above equation to hold is that $\Gamma(q-v(0)+1 / 2)=\infty$, namely, $q-v(0)+1 / 2$ is a non-positive integer. We may take

$$
v(0)=k+\frac{1}{2}
$$

where $k \in \mathbf{N} \cup\{0\}$ and $q \leq k$. Combining (4.15) and (4.16), we obtain

$$
\begin{aligned}
\left\{-\left(v+\frac{3}{2}\right)\right. & \cos \sigma \cos \varepsilon-\sin \sigma \sin \varepsilon\} \\
\times & {\left[\frac{\Gamma\left(q+\frac{3}{2}\right) \Gamma\left(q+\frac{1}{2}\right)}{\Gamma\left(q+v+\frac{3}{2}\right) \Gamma\left(q-v+\frac{1}{2}\right)} F\left(-v, v+1,-q+\frac{1}{2} ; \frac{t_{\varepsilon}+1}{2}\right)\right.} \\
& +\frac{\Gamma\left(q+\frac{3}{2}\right) \Gamma\left(-q-\frac{1}{2}\right)}{\Gamma(-v) \Gamma(v+1)}\left(\frac{t_{\varepsilon}+1}{2}\right)^{q+1 / 2} \\
& \left.\times F\left(q+v+\frac{3}{2}, q-v+\frac{1}{2}, q+\frac{3}{2} ; \frac{t_{\varepsilon}+1}{2}\right)\right] \\
= & \left(v+\frac{3}{2}\right) \cos \sigma \\
\times & \frac{\Gamma\left(q+\frac{3}{2}\right) \Gamma\left(q+\frac{1}{2}\right)}{\Gamma\left(q+v+\frac{5}{2}\right) \Gamma\left(q-v-\frac{1}{2}\right)} F\left(-(v+1), v+2,-q+\frac{1}{2} ; \frac{t_{\varepsilon}+1}{2}\right) \\
& +\frac{\Gamma\left(q+\frac{3}{2}\right) \Gamma\left(-q-\frac{1}{2}\right)}{\Gamma(-v-1) \Gamma(v+2)}\left(\frac{t_{\varepsilon}+1}{2}\right)^{q+1 / 2} \\
& \left.\times F\left(q+v+\frac{5}{2}, q-v-\frac{1}{2}, q+\frac{3}{2} ; \frac{t_{\varepsilon}+1}{2}\right)\right]
\end{aligned}
$$

with $t_{\varepsilon}=\cos (\pi-\varepsilon)$. Note that both sides become small as $\varepsilon \rightarrow+0$. If $q \geq 1$, then dividing the two sides of $(4.17)$ by $\varepsilon^{2 q+1}$, we obtain 


$$
\begin{aligned}
-\{(v+ & \left.\left.\frac{3}{2}\right) \cos \sigma \cos \varepsilon+\sin \sigma \sin \varepsilon\right\} \\
& \times\left\{\frac{(-1)^{k-q+1} \Gamma\left(q+\frac{3}{2}\right) \Gamma\left(q+\frac{1}{2}\right)(k-q) ! \tilde{c}_{k, q, \sigma, 3}(\varepsilon)}{\Gamma(q+k+2)}\right. \\
& \left.+\frac{\Gamma\left(q+\frac{3}{2}\right) \Gamma\left(-q-\frac{1}{2}\right)}{\Gamma\left(-k-\frac{1}{2}\right) \Gamma\left(k+\frac{3}{2}\right) 2^{2 q+1}}+O(\varepsilon)\right\} \\
= & \left(v+q+\frac{3}{2}\right) \cos \sigma \\
& \times\left\{\frac{(-1)^{k-q+2} \Gamma\left(q+\frac{3}{2}\right) \Gamma\left(q+\frac{1}{2}\right)(k+1-q) ! \tilde{c}_{k, q, \sigma, 3}(\varepsilon)}{\Gamma(q+k+3)}\right. \\
& \left.+\frac{\Gamma\left(q+\frac{3}{2}\right) \Gamma\left(-q-\frac{1}{2}\right)}{\Gamma\left(-k-\frac{3}{2}\right) \Gamma\left(k+\frac{5}{2}\right) 2^{2 q+1}}+O(\varepsilon)\right\},
\end{aligned}
$$

where

$$
v=v(\varepsilon)=k+\frac{1}{2}+\tilde{c}_{k, q, \sigma, 3}(\varepsilon) \varepsilon^{2 q+1}+O\left(\varepsilon^{2 q+2}\right) .
$$

Substituting these relation for (4.18), we have

$$
\begin{array}{r}
\left\{\left(v+\frac{3}{2}\right) \cos \sigma \cos \varepsilon+\sin \sigma \sin \varepsilon\right\}\left\{\tilde{c}_{k, q, \sigma, 3}(\varepsilon)-A+O(\varepsilon)\right\} \\
=\left(v+q+\frac{3}{2}\right) \cos \sigma\left\{\frac{k+1-q}{q+k+2} \tilde{c}_{k, q, \sigma, 3}(\varepsilon)-A+O(\varepsilon)\right\},
\end{array}
$$

where

$$
A=\frac{(-1)^{k-q+2} \Gamma\left(-q-\frac{1}{2}\right) \Gamma(q+k+2)}{\Gamma\left(q+\frac{1}{2}\right)(k-q) ! 2^{2 q+1} \pi} .
$$

Here we used the recursion relation (2.13). Applying the recursion formula (2.12) several times and $\Gamma(1 / 2)=\sqrt{\pi}$, we have

$$
\Gamma\left(-m-\frac{1}{2}\right)=(-1)^{m+1} \frac{2^{2(m+1)}(m+1) !}{\{2(m+1)\} !} \sqrt{\pi}, \quad \Gamma\left(m+\frac{1}{2}\right)=\frac{(2 m) !}{2^{2 m} m !} \sqrt{\pi}
$$

for any nonnegative integer $m$. This implies that

$$
A=\frac{2^{2 q+1}(q+1)(q+k+1) !(q !)^{2}}{(k-q) !\{2(q+1)\} !(2 q) ! \pi} .
$$


By (4.19), we have

$$
\begin{gathered}
\left\{\left(v+\frac{3}{2}\right) \cos \sigma \cos \varepsilon+\sin \sigma \sin \varepsilon-\frac{(k+1-q)\left(v+q+\frac{3}{2}\right) \cos \sigma}{q+k+2}\right\} \tilde{c}_{k, q, \sigma, 3}(\varepsilon) \\
=\left\{\left(v+\frac{3}{2}\right) \cos \sigma \cos \varepsilon+\sin \sigma \sin \varepsilon-\left(v+q+\frac{3}{2}\right) \cos \sigma\right\} A+O(\varepsilon)
\end{gathered}
$$

which implies

$$
\begin{aligned}
\tilde{c}_{k, q, \sigma, 3}(\varepsilon) & \left(v+\frac{3}{2}\right) \cos \sigma+\sin \sigma \sin \varepsilon-\left(v+q+\frac{3}{2}\right) \cos \sigma \\
& =\frac{\left(v+\frac{3}{2}\right) \cos \sigma+\sin \sigma \sin \varepsilon-\frac{(k+1-q)\left(v+q+\frac{3}{2}\right) \cos \sigma}{q+k+2}}{(v+2}
\end{aligned}+O(\varepsilon) .
$$

Thus we obtain

$$
\tilde{c}_{k, q, \sigma, 3}=\lim _{\varepsilon \rightarrow+0} \tilde{c}_{k, q, \sigma, 3}(\varepsilon)= \begin{cases}-\frac{2^{2 q+1} q(k+q+1) !(q !)^{2}}{(k-q) !\{(2(q+1)\} !(2 q) ! \pi}, & \sigma \in\left[0, \frac{\pi}{2}\right), \\ \frac{2^{2 q+1}(q+1)(q+k+1) !(q !)^{2}}{(k-q) !\{2(q+1)\} !(2 q) ! \pi}, & \sigma=\frac{\pi}{2}\end{cases}
$$

If $q=0$, then formally we have $\tilde{c}_{k, 0, \sigma, 3}=0$ for $\sigma \in[0, \pi / 2)$ and $\tilde{c}_{k, 0, \sigma, 3} \neq 0$ for $\sigma=\pi / 2$. Thus, the order must be smaller than $\varepsilon$ if $\sigma \in[0, \pi / 2)$ and $q=0$. In this case, (4.17) yields

$$
\begin{gathered}
\left(-\cos \sigma \cos \varepsilon-\frac{\sin \sigma \sin \varepsilon}{v+\frac{3}{2}}\right)\left[\frac{\Gamma\left(\frac{3}{2}\right) \Gamma\left(\frac{1}{2}\right)}{\Gamma\left(v+\frac{3}{2}\right) \Gamma\left(-v+\frac{1}{2}\right)} F\left(-v, v+1,-\frac{1}{2} ; \frac{t_{\varepsilon}+1}{2}\right)\right. \\
\left.+\frac{\Gamma\left(\frac{3}{2}\right) \Gamma\left(-\frac{1}{2}\right)}{\Gamma(-v) \Gamma(v+1)}\left(\frac{t_{\varepsilon}+1}{2}\right)^{1 / 2} F\left(v+\frac{3}{2},-v+\frac{1}{2}, \frac{3}{2} ; \frac{t_{\varepsilon}+1}{2}\right)\right] \\
=\frac{\Gamma\left(\frac{3}{2}\right) \Gamma\left(\frac{1}{2}\right) \cos \sigma}{\Gamma\left(v+\frac{5}{2}\right) \Gamma\left(-v-\frac{1}{2}\right)} F\left(-(v+1), v+2, \frac{1}{2} ; \frac{t_{\varepsilon}+1}{2}\right) \\
+\frac{\Gamma\left(\frac{3}{2}\right) \Gamma\left(-\frac{1}{2}\right) \cos \sigma}{\Gamma(-v-1) \Gamma(v+2)}\left(\frac{t_{\varepsilon}+1}{2}\right)^{1 / 2} F\left(v+\frac{5}{2},-v-\frac{1}{2}, \frac{3}{2} ; \frac{t_{\varepsilon}+1}{2}\right) .
\end{gathered}
$$

and we need more exact calculations.

Now we write $v=k+\frac{1}{2}+v^{*}(\varepsilon)$. In this case, we use the approximation $\cos \varepsilon=1-\varepsilon^{2} / 2+O\left(\varepsilon^{4}\right)$. Since $\Gamma(-v+1 / 2)$ and $\Gamma(-v-1 / 2)$ has a zero of order $v^{*}(\varepsilon)$ at $\varepsilon=0$, we only need 


$$
\begin{aligned}
& F\left(-v, v+1,-\frac{1}{2} ; \frac{t_{\varepsilon}+1}{2}\right)=1+O\left(\varepsilon^{2}\right), \\
& F\left(-(v+1), v+2, \frac{1}{2} ; \frac{t_{\varepsilon}+1}{2}\right)=1+O\left(\varepsilon^{2}\right) .
\end{aligned}
$$

However, for other $F$ 's, we use the following expansions:

$$
\begin{aligned}
F\left(v+\frac{3}{2},-v+\frac{1}{2}, \frac{3}{2} ; \frac{t_{\varepsilon}+1}{2}\right) & =F\left(k+2+v^{*}(\varepsilon),-k-v^{*}(\varepsilon), \frac{3}{2} ; \frac{t_{\varepsilon}+1}{2}\right) \\
& =1-\frac{k(k+2)}{6} \varepsilon^{2}+O\left(\varepsilon^{4}\right) \\
F\left(v+\frac{5}{2},-v-\frac{1}{2}, \frac{3}{2} ; \frac{t_{\varepsilon}+1}{2}\right) & =F\left(k+3+v^{*}(\varepsilon),-k-1 v^{*}(\varepsilon), \frac{3}{2} ; \frac{t_{\varepsilon}+1}{2}\right) \\
& =1-\frac{(k+1)(k+3)}{6} \varepsilon^{2}+O\left(\varepsilon^{4}\right) .
\end{aligned}
$$

Using (2.12) and (2.13), we have

$$
\Gamma\left(v+\frac{3}{2}\right) \Gamma\left(-v+\frac{1}{2}\right)=\left(v+\frac{1}{2}\right) \Gamma\left(v+\frac{1}{2}\right) \Gamma\left(-v+\frac{1}{2}\right)=\frac{\left(v+\frac{1}{2}\right) \pi}{\sin \left(v+\frac{1}{2}\right) \pi} .
$$

Similarly we obtain

$$
\begin{aligned}
& \frac{\Gamma\left(\frac{3}{2}\right) \Gamma\left(\frac{1}{2}\right)}{\Gamma\left(v+\frac{3}{2}\right) \Gamma\left(-v+\frac{1}{2}\right)}=\frac{\sin \left(v+\frac{1}{2}\right) \pi}{2 v+1}, \quad \frac{\Gamma\left(\frac{3}{2}\right) \Gamma\left(-\frac{1}{2}\right)}{\Gamma(-v) \Gamma(v+1)}=\sin v \pi \\
& \frac{\Gamma\left(\frac{3}{2}\right) \Gamma\left(\frac{1}{2}\right)}{\Gamma\left(v+\frac{5}{2}\right) \Gamma\left(-v-\frac{1}{2}\right)}=-\frac{\sin \left(v+\frac{1}{2}\right) \pi}{2 v+3},
\end{aligned}
$$

Then the following part of (4.23) can be calculated as

$$
\begin{aligned}
\left(-\cos \sigma \cos \varepsilon-\frac{\sin \sigma \sin \varepsilon}{v+\frac{3}{2}}\right) \frac{\Gamma\left(\frac{3}{2}\right) \Gamma\left(-\frac{1}{2}\right)}{\Gamma(-v) \Gamma(v+1)} F\left(v+\frac{3}{2},-v+\frac{1}{2}, \frac{3}{2} ; \frac{t_{\varepsilon}+1}{2}\right) \\
\quad-\frac{\Gamma\left(\frac{3}{2}\right) \Gamma\left(-\frac{1}{2}\right) \cos \sigma}{\Gamma(-v-1) \Gamma(v+2)} F\left(v+\frac{5}{2},-v-\frac{1}{2}, \frac{3}{2} ; \frac{t_{\varepsilon}+1}{2}\right) \\
=\left[\operatorname { c o s } \sigma \left(F\left(v+\frac{5}{2},-v-\frac{1}{2}, \frac{3}{2} ; \frac{t_{\varepsilon}+1}{2}\right)\right.\right. \\
\left.\quad-F\left(v+\frac{3}{2},-v+\frac{1}{2}, \frac{3}{2} ; \frac{t_{\varepsilon}+1}{2}\right) \cos \varepsilon\right) \\
\left.\quad-\frac{\sin \sigma \sin \varepsilon}{v+\frac{3}{2}} F\left(v+\frac{3}{2},-v+\frac{1}{2}, \frac{3}{2} ; \frac{t_{\varepsilon}+1}{2}\right)\right] \sin \left(k+\frac{1}{2}+v^{*}(\varepsilon)\right) \pi
\end{aligned}
$$




$$
\begin{aligned}
= & {\left[\operatorname { c o s } \sigma \left\{1-\frac{(k+1)(k+3)}{6} \varepsilon^{2}+O\left(\varepsilon^{4}\right)\right.\right.} \\
& \left.-\left(1-\frac{k(k+2)}{6} \varepsilon^{2}-\frac{1}{2} \varepsilon^{2}+O\left(\varepsilon^{4}\right)\right)\right\} \\
& \left.-\frac{\sin \sigma \sin \varepsilon}{v+\frac{3}{2}}\left(1-\frac{k(k+2)}{6} \varepsilon^{2}+O\left(\varepsilon^{4}\right)\right)\right](-1)^{k} \sin \left(\frac{1}{2}+v^{*}(\varepsilon)\right) \pi \\
= & \left(-\frac{(-1)^{k} \varepsilon \sin \sigma}{k+2}+O\left(\varepsilon^{2}\right)\right)
\end{aligned}
$$

On the other hand, the rest of (4.23) becomes

$$
\begin{aligned}
& \frac{\Gamma\left(\frac{3}{2}\right) \Gamma\left(\frac{1}{2}\right) \cos \sigma}{\Gamma\left(v+\frac{5}{2}\right) \Gamma\left(-v-\frac{1}{2}\right)} F\left(-(v+1), v+2, \frac{1}{2} ; \frac{t_{\varepsilon}+1}{2}\right) \\
& -\left(-\cos \sigma \cos \varepsilon-\frac{\sin \sigma \sin \varepsilon}{v+\frac{3}{2}}\right) \frac{\Gamma\left(\frac{3}{2}\right) \Gamma\left(\frac{1}{2}\right)}{\Gamma\left(v+\frac{3}{2}\right) \Gamma\left(-v+\frac{1}{2}\right)} \\
& \times F\left(-v, v+1,-\frac{1}{2} ; \frac{t_{\varepsilon}+1}{2}\right) \\
& =-\frac{\cos \sigma \sin \left(v+\frac{1}{2}\right) \pi}{2 v+3} F\left(-(v+1), v+2, \frac{1}{2} ; \frac{t_{\varepsilon}+1}{2}\right) \\
& -\left(-\cos \sigma \cos \varepsilon-\frac{\sin \sigma \sin \varepsilon}{v+\frac{3}{2}}\right) \frac{\sin \left(v+\frac{1}{2}\right) \pi}{2 v+1} \\
& \times F\left(-v, v+1,-\frac{1}{2} ; \frac{t_{\varepsilon}+1}{2}\right) \\
& =\cos \sigma\left[\frac{1}{2(k+1)(k+2)}+O(\varepsilon)\right](-1)^{k+1} \sin v^{*} \pi .
\end{aligned}
$$

Substituting the above terms (4.24) and (4.25) for (4.23), we have

$$
\left(\frac{(-1)^{k+1} \varepsilon \sin \sigma}{k+2}+O\left(\varepsilon^{2}\right)\right) \frac{\varepsilon}{2}=\cos \sigma\left[\frac{(-1)^{k+1}}{2(k+1)(k+2)}+O(\varepsilon)\right] \sin v^{*} \pi
$$

Thus we get

$$
\tilde{c}_{k, 0, \sigma, 3}=\lim _{\varepsilon \rightarrow+0} \varepsilon^{-2} v^{*}(\varepsilon)=\frac{(k+1) \tan \sigma}{\pi} .
$$

Finally, as in Section 3, we have

$$
\lambda=v(\varepsilon)^{2}+v-\frac{3}{4}=\left(v(\varepsilon)+\frac{3}{2}\right)\left(v(\varepsilon)-\frac{1}{2}\right)=\left(k+2+v^{*}(\varepsilon)\right)\left(k+v^{*}(\varepsilon)\right) .
$$


Thus the desired constant in Theorem 1.2 is determined by $c_{k, q, \sigma, 3}=$ $2(k+1) \tilde{c}_{k, q, \sigma, 3}$.

The multiplicity of the eigenvalue comes from the construction of eigenfunctions. That is, in (4.8), $m$ varies from 0 to $q$. If $m=0$, we see

$$
\Phi=c_{1} \frac{P_{v}^{-(q+1 / 2)}(\cos \theta)}{\sqrt{\sin \theta}} P_{q}^{0}(\cos \varphi)
$$

and this has a one dimensional space and for each $m \geq 1$, the set of the eigenfunctions in (4.8) is two dimensional. Hence, $1+\sum_{m=1}^{q} 2=2 q+1$ is the total multiplicity.

Remark 4.1. The order of $v^{*}(\varepsilon)$ is also obtained by the following way. Let the eigenvalue $\lambda$ be determined as

$$
\lambda=v(\varepsilon)^{2}+v(\varepsilon)-\frac{3}{4}=\left(v(\varepsilon)+\frac{3}{2}\right)\left(v(\varepsilon)-\frac{1}{2}\right) .
$$

Now take $\xi>0$ so that

$$
\xi^{2}-1=\lambda=\left(v(\varepsilon)+\frac{3}{2}\right)\left(v(\varepsilon)-\frac{1}{2}\right) .
$$

Then it follows that $\xi=k+1+v^{*}(\varepsilon)$. Hence

$$
\Xi(\theta)=\frac{\sin \xi \theta}{\sin \theta}
$$

is a regular solution to

$$
\Lambda \Xi+\lambda \Xi=0
$$

in $\Omega_{\varepsilon} \subset \mathbf{S}^{3}$. We seek the condition of $v^{*}(\varepsilon)$ so that

$$
(\cos \sigma) \Xi^{\prime}(\pi-\varepsilon)+(\sin \sigma) \Xi(\pi-\varepsilon)=0 .
$$

By the direct calculations, we see that

$$
\left(k+1+v^{*}(\varepsilon)\right) \tan (\pi-\varepsilon)=(1-\tan \sigma \tan \varepsilon) \tan \left\{\left(k+1+v^{*}(\varepsilon)\right)(\pi-\varepsilon)\right\}
$$

is the condition for $v^{*}(\varepsilon)$. Expanding the both sides in the Taylor series and we pick up the order up to $\varepsilon^{2}$, we have

$$
\begin{aligned}
-\left(k+1+v^{*}(\varepsilon)\right)\left(\varepsilon+O\left(\varepsilon^{2}\right)\right)= & -(k+1) \varepsilon+\pi v^{*}(\varepsilon)-\tan \sigma \tan \varepsilon \tan (k+1) \varepsilon \\
& +O\left(\varepsilon^{3}+v^{*}(\varepsilon) \varepsilon\right) .
\end{aligned}
$$

Then, the $\varepsilon$ order terms vanish and we obtain

$$
\pi v^{*}(\varepsilon)=(k+1)(\tan \sigma) \varepsilon^{2}+O\left(\varepsilon^{3}\right) .
$$

Thus, we obtain the same order and the same coefficient as above. 
Acknowledgment. The authors thank Professor Catherine Bandle for valuable private communication.

\section{REFERENCES}

[1] F. E. BAGINSKI, Ordering the zeros of Legendre functions $P_{v}^{m}\left(z_{0}\right)$ when considered as a function of $v$, J. Math. Anal. Appl. 147 (1990), 269-308.

[2] F. E. Baginski, Comparison theorems for the $v$-zeros of Legendre functions $P_{v}^{m}\left(z_{0}\right)$ when $-1<z_{0}<1$, Proc. Amer. Math. Soc. 111 (1991), 395-402.

[ 3 ] C. Bandle and R. Benguria, The Brezis-Nirenberg problem on $\mathbf{S}^{3}$, J. Differential Equations 178 (2002), 264-279.

[4] C. Bandle, A. Brillard and M. Flucher, Green's function, harmonic transplantation, and the best Sobolev constant in spaces of constant curvature, Trans. Amer. Math. Soc. 350 (1998), 1103-1128.

[ 5 ] C. Bandle, Y. Kabeya and H. Ninomiya, Imperfect bifurcations in nonlinear elliptic equations on spherical caps, Comm. Pure Appl. Anal. 10 (2010), 1189-1208.

[ 6 ] C. Bandle, Y. Kabeya and H. Ninomiya, Bifurcating solution of a nolinear elliptic Neumann problem on large spherical caps, preprint.

[7] C. Bandle and L. A. Peletier, Best Sobolev constants and Emden equations for the critical exponent in $\mathbf{S}^{\mathbf{3}}$, Math. Ann. 313 (1999), 83-93.

[ 8 ] R. Beals and R. Wong, Special functions: A graduate text, Cambridge studies in advanced mathematics 126, Cambridge UP, Cambridge, 2010.

[9] H. Brezis and L. A. Peletier, Elliptic equations with critical exponent on spherical caps of $\mathbf{S}^{3}$, J. Anal. Math. 98 (2006), 279-316.

[10] G. Courtors, Spectrum of manifolds with holes, J. Funct. Anal. 134 (1995), 194-221.

[11] A. KosaKa, Bifurcations of solutions to semilinear elliptic problems on $\mathbf{S}^{\mathbf{2}}$ with a small hole, to appear in Sci. Math. Jpn.

[12] M. Lanza De Cristoforis, Simple Neumann eigenvalues for the Laplace operator in a domain with a small hole, Rev. Mat. Complut. 25 (2012), 369-412.

[13] H. M. Macdonald, Zeros of the spherical harmonics $P_{n}^{m}(\mu)$ considered as a function of $n$, Proc. London Math. Soc. 31 (1899), 264-278.

[14] S. Moriguchi, K. Udagawa and S. Hitotsumatsu, Mathematical formula III, Iwanami Shoten, Tokyo, 1960 (in Japanese).

[15] W.-M. NI, The mathematics of diffusion, SIAM, Philadelphia, 2011.

[16] S. Ozawa, Singular variations of domains and eigenvalues of the Laplacian, Duke Math. J. 48 (1981), 767-778.

[17] S. Ozawa, Electrostatic capacity and eigenvalues of the Laplacian, J. Fac. Sci. Univ. Tokyo Sect. IA Math. 30 (1983), 53-62.

[18] S. Ozawa, An asymptotic formula for the eigenvalues of the Laplacian in a three dimensional domain with a small hole, J. Fac. Sci. Univ. Tokyo Sect. IA Math. 30 (1983), 243-247.

[19] S. OzAwa, Asymptotic property of an eigenfunction of the Laplacian under singular variation of domains - the Neumann condition-, Osaka Math. J. 22 (1985), 639-655.

[20] H. PrüFeR, Neue Herleitung der Sturm-Liouvilleschen Reihenentwicklung stetiger Functionen, Math. Ann. 95 (1926), 499-518.

[21] N. Shimakura, The partial differential operators of elliptic type, AMS, Providence, 1992.

[22] E. C. Tichmarsh, Eigenfunction expansions associated with partial differential equations (V), Proc. London Math. Soc. (3) 5 (1955), 1-21.

[23] E. C. Tichmarsh, Eigenfunction expansion, Part 1, Oxford Univ. Press, Oxford, 1962. 
[24] K. Yosida, The method of solving differential equations, 2nd ed., Iwanami Shoten, Tokyo, 1978 (in Japanese).

Yoshitsugu Kabeya

Department of Mathematical Sciences

Osaka Prefecture University

Gakuencho, SakaI, 599-8531

JAPAN

E-mail: kabeya@ms.osakafu-u.ac.jp

Tatsuki Kawakami

Department of Mathematical Sciences

Osaka Prefecture University

GAKUENCHO, SAKaI, 599-8531

JAPAN

E-mail: kawakami@ms.osakafu-u.ac.jp

Atsushi Kosaka

Advanced Mathematical Institute

OSAKA City UNIVERSITY

SugImotocho, OsaKa, 558-8585

JAPAN

E-mail: kosaka@sci.osaka-cu.ac.jp

Hirokazu Ninomiya

SCHOOL OF INTERdisciplinary Mathematical SCIENCES

MeIJI UNIVERSITY

NAKANO, TOKYO, 164-8525

JAPAN

E-mail: ninomiya@math.meiji.ac.jp 